\title{
A chronic thigh mass in a 69-year-old man
}

\author{
Simon A. S. Beggs • Awatif Al-Nafussi • \\ C. Michael Lambert • Daniel Porter • J. T. Patton
}

Published online: 18 June 2010

(C) ISS 2010

\section{Question}

A 69-year-old man presented with a solitary, painless swelling on the lateral aspect of his left thigh that had been present, unchanged for 10 years. His medical history included chronic obstructive pulmonary disease, hypertension and bilateral knee osteoarthritis.
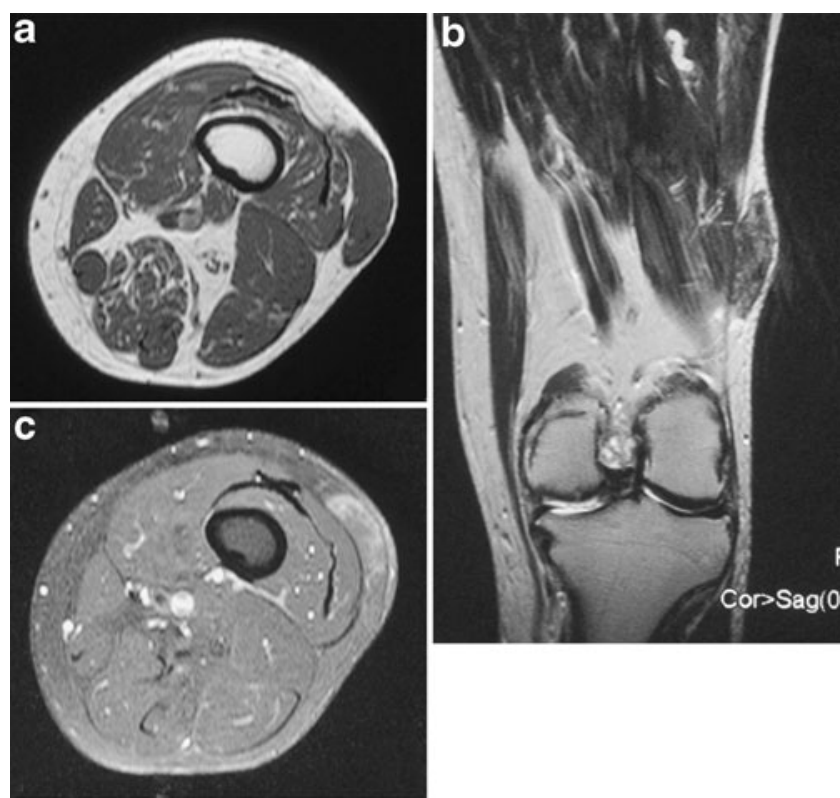

The diagnosis can be found at doi: 10.1007/s00256-010-0973-6.

S. A. S. Beggs $(\bowtie)$

Edinburgh University College

of Medicine and Veterinary Medicine,

The Chancellor's Building 2nd Floor, 49 Little France Crescent,

Edinburgh EH16 4SB, UK

e-mail: beggs1@googlemail.com

\section{A. Al-Nafussi}

Department of Pathology, Royal Infirmary,

Edinburgh EH16 4SA, UK

\section{M. Lambert}

Department of Rheumatology, Western General Hospital,

Edinburgh EH4 2XU, UK

\section{Porter $\cdot$ J. T. Patton}

Department of Orthopaedics, Royal Infirmary,

Edinburgh EH16 4SA, UK

Fig. 1 Magnetic resonance imaging of the thigh, including a axial T1-weighted, b coronal T2-weighted and c axial fat-suppressed T1weighted images after gadolinium administration, was followed by ultrasound-guided core biopsy 